\title{
PRUEBAS DE TOXICIDAD CON ELUTRIADOS DE SEDIMENTO MARINO DE LAS BAHÍAS LOS CHIMUS, TORTUGAS Y CASMA EN PERÚ EMPLEANDO LAS MICROALGAS Isochrysis galbana Y Nannochloropsis oceanica
}

\section{TOXICITY TESTING WITH MARINE SEDIMENT ELUTRIATES FROM LOS CHIMUS, TORTUGAS AND CASMA BAYS IN PERU USING THE MICROALGAE Isochrysis galbana AND Nannochloropsis oceanica}

\author{
Haydeé López ${ }^{1,2}$, María Cristina Miglio ${ }^{1,3}$ y Christian Paredes ${ }^{4}$
}

\begin{abstract}
Resumen
Se realizaron pruebas de toxicidad con elutriados de sedimentos marinos provenientes de las bahías Los Chimus, Tortugas y Casma, en Perú, empleando las microalgas marinas Isochrysis galbana y Nannochloropsis oceanica. Las muestras de sedimento marino se colectaron en los meses de marzo y agosto de 2016. Estas pruebas de toxicidad se realizaron siguiendo los protocolos estandarizados, mediante pruebas estáticas a $96 \mathrm{~h}$ de exposición. Previamente, se determinó la sensibilidad de ambas cepas de microalgas al sulfato de cobre $\left(\mathrm{CuSO}_{4} \cdot 5 \mathrm{H}_{2} \mathrm{O}\right)$ como sustancia de referencia. La Concentración de Inhibición media $\left(\mathrm{CI}_{50}\right)$ obtenida en este ensayo demostró que I. galbana fue más sensible que $N$. oceanica. Los elutriados de los sedimentos marinos, provenientes de las zonas de estudio, presentaron diferentes niveles de toxicidad, tales como "no toxicidad" $\left(\mathrm{CI}_{50}=90 \%-100 \%\right)$, "moderada toxicidad" $\left(\mathrm{CI}_{50}=59 \%-81 \%\right)$ y "alta toxicidad" $\left(\mathrm{CI}_{50}<59 \%\right)$ al emplear la microalga I. galbana; mientras que, para el caso de $N$. oceanica solo se presentó el de "no toxicidad" $\left(\mathrm{CI}_{50}=90 \%-100 \%\right)$ frente a las mismas muestras de elutriados de sedimentos. Estas pruebas de toxicidad demuestran que la microalga I. galbana detecta diferentes niveles de toxicidad, por lo que se podría emplear para evaluar ambientalmente muestras de sedimento marino como complemento a pruebas químicas, ya que no se discrimina el origen de la toxicidad.
\end{abstract}

Palabras clave: $\mathrm{CI}_{50}$, Isochrysis galbana, Nannochloropsis oceanica, prueba de toxicidad, elutriados de sedimentos marinos, sulfato de cobre.

\begin{abstract}
Toxicity tests were carried out on marine sediment elutriates from Los Chimus, Tortugas, and Casma bays in Peru, using the marine microalgae Isochrysis galbana and Nannochloropsis oceanica. The marine sediment samples were collected in the months of March and August 2016. These toxicity tests were performed following standardized protocols, using static tests at $96 \mathrm{~h}$ of exposure. Previously, the sensitivity of both strains of microalgae was determined using copper sulfate $\left(\mathrm{CuSO}_{4} \cdot 5 \mathrm{H}_{2} \mathrm{O}\right)$ as a reference substance. The mean Inhibition Concentration ( $\mathrm{IC}_{50}$ ) obtained in this test showed that I. galbana was more sensitive than N. oceanica. Marine sediment elutriates from the study areas showed different levels of toxicity, such as "non-toxicity" $\left(\mathrm{IC}_{50}=90 \%-100 \%\right)$, "moderate toxicity" $\left(\mathrm{IC}_{50}=59 \%-81 \%\right)$, and "high toxicity" ( $\left.\mathrm{IC}_{50}<59 \%\right)$ when using the microalgae I. galbana; while, in the case of $N$. oceanica, only "non-toxicity" $\left(\mathrm{IC}_{50}=90 \%-100 \%\right)$ was found for the same sediment elutriate samples. These toxicity tests show that the I. galbana microalgae detects different levels of toxicity, which is why it could be used to environmentally evaluate marine sediment samples as a complement to chemical tests since the origin of toxicity is not discriminated.
\end{abstract}

Key words: $\mathrm{IC}_{50}$, Isochrysis galbana, Nannochloropsis oceanica, toxicity test, elutriate of marine sediment, copper sulphate.

\section{Introducción}

Las fuentes de contaminantes, principalmente de origen antrópico como las descargas de zonas altamente urbanizadas, drenaje urbano y escorrentía de carreteras, conllevan a la modificación del hábitat y la extinción local temporal y/o definitiva de muchos organismos considerados recursos y otros sin fines comerciales, causando daños severos en la salud del ecosistema y la biodiversidad marina (Uribe et al., 2019).
Los contaminantes que ingresan al medio acuático permanecen suspendidos en la columna de agua, se incorporan a la biota acuática o se depositan sobre el fondo en los sedimentos donde permanecen en contacto con organismos bentónicos haciéndolos disponibles a la columna de agua, produciendo así riesgos potenciales para la biota acuática (Valdés \& Castillo, 2014).

Se consideran a los sedimentos marinos como un importante sumidero para sustancias tóxicas, 
convirtiéndolos en fuentes de contaminación que pueden alterar a las comunidades biológicas naturales circundantes y las cadenas tróficas (Alzahrani et al., 2018).

MacIntosh et al. (2021) manifiestan que, las pruebas químicas de detección de contaminantes en sedimentos deberían ser complementadas con pruebas de toxicidad para la evaluación de calidad de los sedimentos. Simpson et al. (2005) consideran que, en estudios de evaluación de seguimiento de la toxicidad marina, la parte superior de 0 a $5 \mathrm{~cm}$ es el horizonte de interés debido a que la tasa de sedimentación en los cuerpos de agua típicamente varía de milímetros a 1-2 cm/año.

Al emplear las pruebas de toxicidad, es necesario contar con pruebas confiables y reproducibles para asegurar la exactitud de los resultados (Díaz et al., 2014). Por tal motivo, la USEPA (1994) recomienda compuestos tóxicos de referencia para estandarizar las pruebas de toxicidad, como cloruro de sodio $(\mathrm{NaCl})$, cloruro de potasio $(\mathrm{KCl})$, cloruro de cadmio $\left(\mathrm{CdCl}_{2}\right)$, sulfato de cobre $\left(\mathrm{CuSO}_{4}\right)$, dodecil sulfato de sodio (SDS) y dicromato de potasio $\left(\mathrm{K}_{2} \mathrm{Cr}_{2} \mathrm{O}_{7}\right)$. Visviki \& Rachlin (1992) mencionan que el tóxico de referencia sulfato de cobre es un nutriente traza de importancia en el metabolismo algal que permite el transporte electrónico; además, es cofactor del componente de muchas enzimas.

En estas pruebas de toxicidad comúnmente son usadas especies de la red trófica acuática como microalgas (Otero et al., 2013), artrópodos (Rudolph et $a l ., 2014$ ), peces (Regidor \& Guzmán, 2015), entre otros. De éstos, las microalgas son excelentes organismos para realizar ensayos de toxicidad, ya que su manejo en cultivo es simple. Además, estos bioensayos son rápidos y de bajo costo en comparación con ensayos con peces e invertebrados (Wong \& Dixon, 1995). La ventaja del uso de microalgas depende del crecimiento, manipulación y grado de sensibilidad (Otero et al., 2013).

A nivel mundial, las pruebas de toxicidad en sedimentos empleando microalgas es mínimo, sólo se tienen referencias del empleo de Dunaliella tertiolecta y Dunaliella salina (Rudolph et al., 2011). En Alemania y Países Bajos se utilizaron las microalgas Phaeodactylum tricornutum y

Desmodesmus subspicatus (Manz et al., 2007).

Trenfield et al. (2015) señalan que, dentro de sus investigaciones, han realizado pruebas de toxicidad con aluminio (Al), galio (Ga) y molibdeno (Mo), metales clave de descarga de refinería de aluminio, empleando a la microalga Isochrysis galbana. Esta misma microalga se empleó para evaluar la toxicidad y el riesgo ambiental que los filtros ultravioletas, ampliamente utilizados en cosmética y como aditivos plásticos, ocasionan en el medio marino (Giraldo et al., 2017). De igual manera, esta microalga ha sido empleada para la evaluación de la calidad de sedimentos contaminados por productos farmacéuticos (Maranho et al., 2015).

Hampel et al. (2001) realizaron ensayos de toxicidad con Nannochloropsis gaditana empleando sulfonato de alquibenceno lineal (LAS), que es un producto químico orgánico sintético utilizado en la elaboración de detergentes, productos de limpieza, así como en la industria textil, alimentos y minería. Para el caso de Nannochloropsis oculata se realizaron bioensayos con solución estándar de hidrocarburo a diferentes concentraciones observando efectos negativos sobre la eficiencia fotosintética de esta especie (Velázquez, 2010). Hasta el momento no hay reportes del uso de la microalga $N$ oceanica para pruebas de toxicidad en ninguna matriz ambiental.

En el Perú, los ensayos de toxicidad con microalgas marinas han sido básicamente para ensayos con sustancias de referencia como cadmio (Vera et al., 2001), cromo (Alayo et al., 2004) y bario (Paredes, 2018), siendo hasta el momento inexistente una evidencia de su uso para la evaluación de sedimentos marinos.

García et al. (2018) mencionan que la calidad ambiental de la Región Áncash está influenciada por los continuos vertimientos de aguas de uso doméstico, aguas de escorrentía agrícola, y ocasionalmente industrial, y el pasivo ambiental de más de 50 años. Estas fuentes de contaminación tienen un impacto negativo sobre el ecosistema marino, las actividades económicas y la salud humana.

Además, Uribe et al. (2019) mencionan que la bahía Los Chimus presenta alta presión por parte del cultivo suspendido del bivalvo Argopecten purpuratus "concha de abanico", el cual se ha extendido en toda la bahía. (García, 2016) menciona que esta bahía ha sido caracterizada por presentar altos niveles de extracción y desembarque de recursos marinos (artesanales) y por tener los mayores bancos naturales de diversos invertebrados marinos en la región. En esta zona existen bancos naturales de A.purpuratus y Romaleon setosum "cangrejo peludo"; también áreas de Stramonita chocolata "caracol negro", áreas acuícolas y áreas propuestas para maricultura (IMARPE, 2006).

Durante este estudio se desarrollaron distintos enfoques de investigación, siendo uno de ellos el empleo de Argopecten purpuratus, organismo bentónico. En este sentido, buscando otra alternativa para realizar pruebas de toxicidad con elutriado de sedimento marino, se decidió emplear las microalgas I. galbana y $N$. oceanica ya que se quería conocer los efectos de la suspensión de contaminantes que, como producto de la remoción del sedimento, podrían ser liberados a la fase acuosa y alterar la calidad del agua superficial.

Al ser las zonas de estudio de aprovechamiento para la acuicultura y con potencial desarrollo para esta actividad, es importante conocer su situación 
ambiental. Cabe mencionar, que actualmente en el Perú no existen normas nacionales, directrices, protocolos, ni guías u otro documento para la evaluación de calidad de sedimento marino, esta investigación constituye un primer paso para la evaluación de elutriados de sedimentos marinos en Perú. Por tales motivos, el objetivo fue analizar la toxicidad del sedimento marino de las bahías Los Chimus, Tortugas y Casma empleando las microalgas I. galbana y N. oceanica durante el año 2016.

\section{Materiales y métodos}

Área de estudio

Las bahías Los Chimus, Tortugas y Casma se encuentran ubicadas en el Departamento de Ancash, Provincias de Casma y Huarmey, entre los $9^{\circ} 28^{\prime} 01^{\prime \prime} \mathrm{S}$ y $\operatorname{los} 78^{\circ} 19^{\prime} 01^{\prime \prime}$ O. Se colectaron muestras de sedimento en ocho estaciones en el mes de marzo y en 10 estaciones en el mes de agosto de 2016 (Tabla 1). Las estaciones de muestreo se ubicaron en zonas litorales cercanas a la costa hasta los $30 \mathrm{~m}$ de profundidad, aproximadamente (Figura 1).

El presente estudio se realizó con el apoyo del Instituto del Mar del Perú (IMARPE), quienes muestrearon 36 estaciones en total, de las cuales se escogieron 14 estaciones para el estudio de pruebas de toxicidad con microalgas, las mismas que se ubicaron en zonas litorales cercanas a la costa, en zonas de cultivo de "concha de abanico", estaciones cercanas a desembocaduras de ríos y otras zonas de actividades económicas (desembarcaderos, centros poblados, industrias pesqueras, entre otros). Las muestras se colectaron con una draga Van Veen de $0.05 \mathrm{~m}^{2}$ de área de mordida a profundidades de entre 4 a $30 \mathrm{~m}$. Cada muestra de sedimento fue colocada en bolsas plásticas que fueron guardadas en envases de polietileno rotulados y conservadas a $4{ }^{\circ} \mathrm{C}$, manteniendo la cadena de frio hasta su utilización en las pruebas de toxicidad (USEPA \& USACE, 1998).

Preparación de elutriado de sedimento marino

El elutriado de sedimento marino se preparó de acuerdo al procedimiento de la USEPA (2002). Se mezclaron $100 \mathrm{~g}$ de sedimento con agua de mar filtrada en una proporción de 1:4. Después de una hora de agitación mecánica de la solución, se dejó reposar por 24 h a $4{ }^{\circ} \mathrm{C}$; luego, se retiró cuidadosamente el sobrenadante sin perturbar el material sedimentado y se obtuvo el elutriado que se centrifugó a 2500 RPM por $3 \mathrm{~min}$; posteriormente se filtró el elutriado con membranas de fibra de vidrio de una micra para separar totalmente la fase líquida de los residuos de sedimentos.

Cultivo de microalgas marinas

El cultivo de las microalgas I. galbana y $N$. oceanica lo desarrolló el personal del Laboratorio de Microalgas del Área de Cultivos Marinos del IMARPE, Callao, siguiendo la metodología de Ynga \& Niño (2019). Este cultivo se realizó en matraces de 1 litro en condiciones controladas para cada especie; siendo, para Isochrysis galbana, $20.3 \pm 1.2{ }^{\circ} \mathrm{C}, \quad \mathrm{pH}=7.9 \pm 0.5$, $\mathrm{S}=35.9 \pm 0.5 \%$, y para Nannochloropsis oceanica, $20.3 \pm 1.0{ }^{\circ} \mathrm{C}, \mathrm{pH}=7.9 \pm 0.37, \mathrm{~S}=36.2 \pm 0.36 \%$. La fase exponencial de los cultivos de microalgas se logró después de 3 a 6 días en medio Guillard F/2 modificado.

Tabla 1. Caracterización del sedimento marino de las estaciones de muestreo en las bahías Los Chimus, Tortugas y Casma en Ancash (Perú) durante marzo y agosto de 2016.

\begin{tabular}{|c|c|c|}
\hline 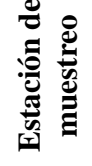 & $\begin{array}{l}\text { Marzo/ } \\
\text { Agosto }\end{array}$ & Características de la zona \\
\hline E1 & $\mathrm{X} /-$ & $\begin{array}{l}\text { A } 2 \mathrm{Km} \text { de la desembocadura del río } \\
\text { Nepeña, margen del río es área de uso } \\
\text { agrícola, a } 3.0 \mathrm{Km} \text { de una planta pesquera }\end{array}$ \\
\hline E2 & $\mathrm{X} / \mathrm{X}$ & $\begin{array}{l}\text { Concesión acuícola de } \\
\text { purpuratus } \\
\text { metálica }\end{array}$ \\
\hline E3 & $-/ X$ & $\begin{array}{l}\text { Concesión acuícola de Argopecten } \\
\text { purpuratus, a } 2.0 \mathrm{Km} \text { de concesión } \\
\text { minera no metálica }\end{array}$ \\
\hline E4 & $-/ X$ & $\begin{array}{l}\text { Concesión acuícola de } \text { Argopecten } \\
\text { purpuratus, a } 4.0 \mathrm{Km} \\
\begin{array}{l}\text { desembocadura del río Nepeña } \\
\text { de }\end{array}\end{array}$ \\
\hline E5 & $-/ X$ & $\begin{array}{l}\text { Concesión acuícola de Argopecten } \\
\text { purpuratus }\end{array}$ \\
\hline E6 & $-/ X$ & $\begin{array}{l}\text { Concesión acuícola de Argopecten } \\
\text { purpuratus }\end{array}$ \\
\hline E7 & $-/ X$ & $\begin{array}{l}\text { Concesión acuícola de Argopecten } \\
\text { purpuratus }\end{array}$ \\
\hline E8 & $-/ X$ & $\begin{array}{l}\text { Concesión acuícola de Argopecten } \\
\text { purpuratus, a } 1.0 \mathrm{Km} \text { de casco urbano }\end{array}$ \\
\hline E9 & $\mathrm{X} / \mathrm{X}$ & $\begin{array}{l}\text { Concesión acuícola de Argopecten } \\
\text { purpuratus, a } 1.0 \mathrm{Km} \text { de casco urbano }\end{array}$ \\
\hline E10 & $\mathrm{X} / \mathrm{X}$ & $\begin{array}{l}\text { Concesión acuícola de Argopecten } \\
\text { purpuratus, a } 1.5 \mathrm{Km} \text { de concesión } \\
\text { minera no metálica }\end{array}$ \\
\hline E11 & $\mathrm{X} /-$ & $\begin{array}{l}\text { A } 2.5 \mathrm{~m} \text { de concesión minera no metálica } \\
\text { y } 4 \mathrm{Km} \text { de la desembocadura de río } \\
\text { Casma }\end{array}$ \\
\hline E12 & $\mathrm{X} /-$ & $\begin{array}{l}\text { A } 1.0 \mathrm{Km} \text { de la desembocadura del río } \\
\text { Casma, al margen del río hay centros } \\
\text { poblados y áreas de uso agrícola, a } 5.0 \\
\mathrm{Km} \text { de una planta pesquera }\end{array}$ \\
\hline E13 & $\mathrm{X} /-$ & $\begin{array}{l}\text { A } 3.5 \mathrm{Km} \text { de concesión minera no } \\
\text { metálica y } 4.0 \mathrm{Km} \text { de la desembocadura } \\
\text { de río Casma }\end{array}$ \\
\hline E14 & $\mathrm{X} / \mathrm{X}$ & $\begin{array}{l}\text { A } 4.0 \mathrm{Km} \text { de la desembocadura del río } \\
\text { Casma, al margen del río hay centros } \\
\text { poblados y áreas de uso agrícola }\end{array}$ \\
\hline
\end{tabular}

La aclimatación de las microalgas se realizó por un lapso de 2-4 h en el Laboratorio de Ecotoxicología Acuática del IMARPE, dentro de una Cámara de iluminación LED de $1.5 \mathrm{~m}^{2}$ de área con una intensidad de (2 $016 \pm 359$ lux $)$, y a una temperatura del ambiente de $20.2 \pm 1.1^{\circ} \mathrm{C}$. Luego de ese tiempo, se procedió a realizar las pruebas de toxicidad. 

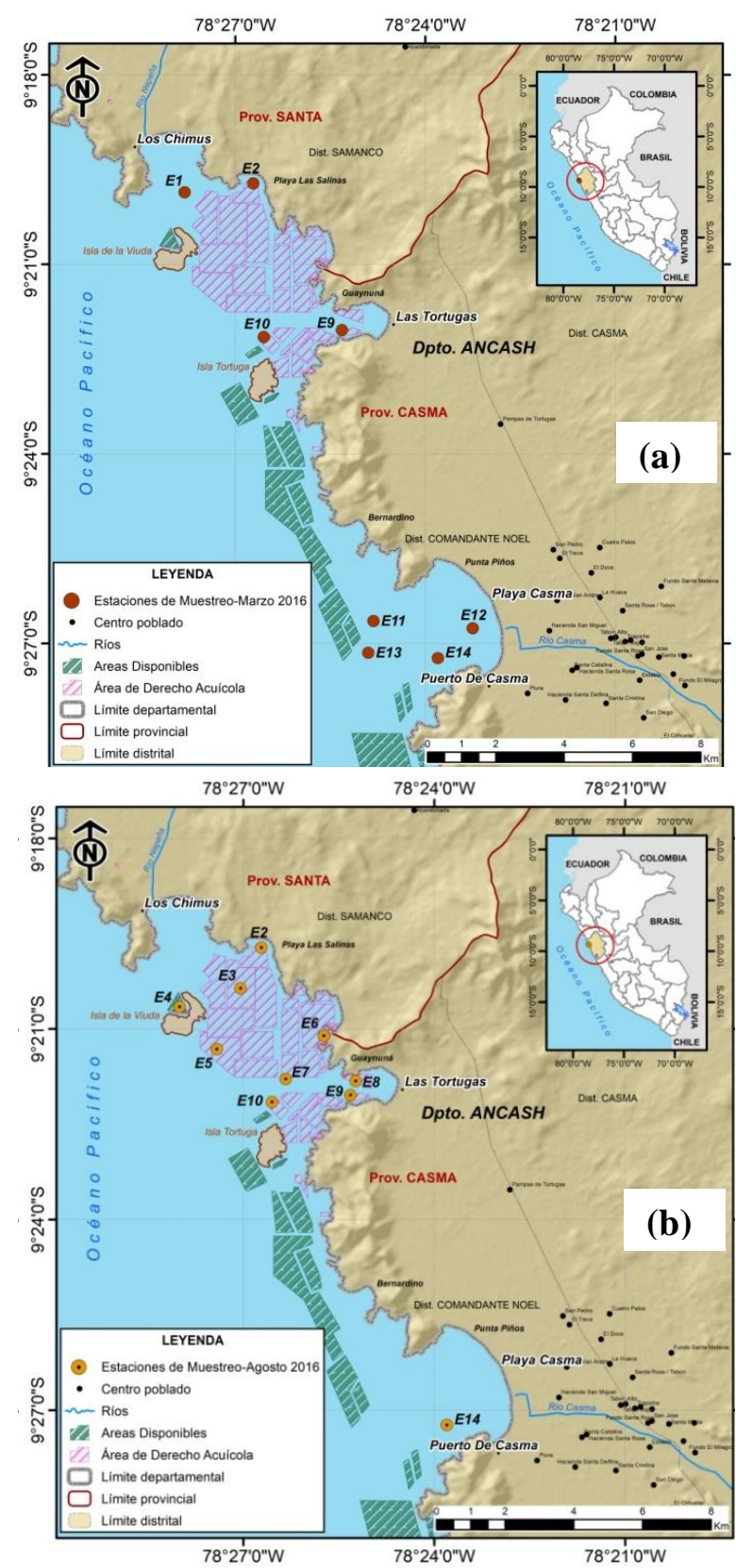

Figura 1. Ubicación de las estaciones de muestreo en las bahías Los Chimus, Tortugas y Casma en Áncash (Perú). (a) marzo 2016 y (b) agosto 2016.

\section{Pruebas de sensibilidad de microalgas}

El tóxico de referencia para probar la sensibilidad de las microalgas fue el sulfato de cobre pentahidratado $\left(\mathrm{CuSO}_{4} \cdot 5 \mathrm{H}_{2} \mathrm{O}\right)$. La prueba de toxicidad fue subletal (USEPA, 1992). Las concentraciones a las que se expusieron las microalgas por 96 horas, fueron: 2.0 $\mathrm{mg} / \mathrm{l}, \quad 1.0 \mathrm{mg} / \mathrm{l}, \quad 0.5 \mathrm{mg} / \mathrm{l}, \quad 0.25 \mathrm{~m} / \mathrm{l}, \quad 0.125 \mathrm{mg} / \mathrm{l}$, considerando un factor de dilución de 0.5 , más un control negativo, con cuatro réplicas por concentración, con un diseño experimental de Diseño de Bloques Completamente Aleatorizado (DBCA) 6x4, siguiendo la metodología propuesta por la USEPA \& USACE (1998) y la USEPA (2012). Las microalgas fueron cultivadas con el medio de cultivo Guillard F/2 modificado a $21^{\circ} \mathrm{C} \pm 1$ y luz continua de 2000 lux.

Tabla 2. Condiciones ambientales utilizadas en las pruebas definitivas o de multiconcentración empleando Isochrysis galbana y Nannochloropsis oceanica.

\begin{tabular}{|c|c|}
\hline PARÁMETROS & CONDICIONES \\
\hline Tipo de prueba & Estática \\
\hline Duración de la prueba & $96 \mathrm{~h}$ \\
\hline Temperatura & $21-23{ }^{\circ} \mathrm{C}$ \\
\hline Salinidad & $34-36 \%$ \\
\hline Iluminación & $\begin{array}{l}\text { Artificial, controlada con luces } \\
\text { LED, } 2500 \text { lux }\end{array}$ \\
\hline Fotoperiodo & $24 \mathrm{~h} \mathrm{luz}$ \\
\hline Aireación & $\begin{array}{l}\text { Agitación manual periódica } \\
\text { durante toda la prueba. }\end{array}$ \\
\hline Recipientes de prueba & $\begin{array}{l}\text { Matraces Erlenmeyer de } 250 \\
\mathrm{ml}\end{array}$ \\
\hline Volumen de prueba & $50 \mathrm{ml}$ \\
\hline $\begin{array}{l}\text { Edad del cultivo al } \\
\text { inóculo }\end{array}$ & $\begin{array}{l}3-6 \text { días (fase exponencial de } \\
\text { crecimiento) }\end{array}$ \\
\hline Agua de dilución & $\begin{array}{l}\text { Agua de mar tratada } \\
\text { (Laboratorios de cultivos } \\
\text { marinos de IMARPE-Callao) }\end{array}$ \\
\hline $\begin{array}{l}\text { Cantidad de nutrientes } \\
\text { por matraz }\end{array}$ & $\begin{array}{l}52 \mu 1 \text { de medio Guillard " } \mathrm{f} / 2 \text { " } \\
\text { modificado }\end{array}$ \\
\hline $\begin{array}{l}\text { Volumen de microalgas } \\
\text { por matraz }\end{array}$ & $2 \mathrm{ml}$ \\
\hline $\begin{array}{l}\text { Densidad celular del } \\
\text { inóculo por matraz }\end{array}$ & $\begin{array}{l}10000 \text { células/ml (I. galbana) } \\
250000 \quad \text { células } / \mathrm{ml} \\
(N . \text { oceanica })\end{array}$ \\
\hline $\begin{array}{l}\text { Número } \\
\text { Concentraciones }\end{array}$ & $\begin{array}{l}\text { Dos concentraciones (prueba } \\
\text { preliminar 0/100), cinco } \\
\text { concentraciones en serie } \\
\text { geométrica (prueba definitiva } \\
\text { y de sensibilidad). }\end{array}$ \\
\hline $\begin{array}{l}\text { Réplicas } \\
\text { concentración }\end{array}$ & Cuatro \\
\hline Efecto medido & $\begin{array}{l}\text { Inhibición de crecimiento } \\
\text { poblacional, medida como } \\
\text { densidad celular }\end{array}$ \\
\hline $\begin{array}{l}\text { Periodicidad de las } \\
\text { observaciones }\end{array}$ & Cada $24 \mathrm{~h}$ \\
\hline $\begin{array}{llr}\text { Criterio } & & \text { de } \\
\text { aceptabilidad } & \text { de } & \text { la } \\
\text { prueba } & & \end{array}$ & $\begin{array}{l}\text { La densidad celular en el } \\
\text { control negativo al finalizar la } \\
\text { prueba }(96 \mathrm{~h}) \text { debe ser } \geq 1 \times 10^{6} \\
\text { células } / \mathrm{ml}\end{array}$ \\
\hline $\begin{array}{l}\text { Coeficiente de variación } \\
\text { en los conteos de } \\
\text { resultado final }\end{array}$ & $\begin{array}{l}\leq 20 \text { por ciento entre réplicas } \\
\text { de un mismo tratamiento } \mathrm{CI}_{50}- \\
96 \mathrm{~h}\end{array}$ \\
\hline Control positivo & $\begin{array}{l}\mathrm{Cu}(\mathrm{II}) \text {, a partir de una solución } \\
\text { de } \mathrm{CuSO}_{4} \cdot 5 \mathrm{H}_{2} \mathrm{O}\end{array}$ \\
\hline
\end{tabular}

Pruebas de toxicidad con elutriado de sedimento $\underline{\text { marino }}$

Las pruebas de toxicidad siguieron las pautas recomendadas de la metodología propuesta por la USEPA (2002), siguiendo las condiciones que se indican en la Tabla 2. Se desarrollaron inicialmente pruebas preliminares (0/100) sometiendo las 
microalgas a concentraciones del $100 \%$ del elutriado y un control negativo sin elutriado, con el fin de que, aquellas muestras que presentaron diferencias significativas $(\mathrm{p}<0.05)$, prosigan a una prueba de multiconcentración.

La densidad celular de microalgas (células/ml) fue determinada mediante recuento de células con cámara de Neubauer y microscopio compuesto.

Análisis estadístico

Los resultados de las pruebas preliminares $(0 / 100)$ se sometieron a una prueba $\mathrm{T}$ de Student, mediante el programa Minitab 15, hallándose las diferencias significativas $\mathrm{p}<0.05$ a las $96 \mathrm{~h}$ entre el control negativo ( $0 \%$ elutriado) y la concentración máxima (100\% de elutriado), para seleccionar qué muestras de sedimento pasarían a las pruebas definitivas o multiconcentración.

Los resultados de las pruebas definitivas o multiconcentración fueron sometidos a la prueba de normalidad de Shapiro-Wilk (S-W) y homogeneidad de varianzas de Levene's. Las diferencias entre cada tratamiento y réplicas a las $96 \mathrm{~h}$ fueron evaluadas con la prueba de Kruskal-Wallis $(\mathrm{K}-\mathrm{W})$ por presentar valores no paramétricos $\mathrm{p}<0.05$ (Zar, 1996). Todos los cálculos estadísticos fueron analizados con el paquete SPSS v21.

Determinación de la Concentración de inhibición media $\left(\mathrm{CI}_{50}\right)$

Para el cálculo del porcentaje de $\mathrm{CI}_{50}$ se utilizó el programa informático ICp (Inhibiting concentration for a specified percent effect) recomendado por la USEPA (1992), donde se realiza una estimación usando el método de interpolación lineal, que compara el control negativo con el valor que reduce el $50 \%$ de la densidad microalgal durante la prueba de inhibición de crecimiento.

Toxicidad del elutriado de sedimento marino

Luego de obtener los porcentajes de $\mathrm{CI}_{50}$ en las muestras evaluadas, se determinó la toxicidad de sedimento marino elutriado siguiendo la metodología de propuesta por Bay et al. (2014) (Tabla 3).

Tabla 3. Toxicidad de elutriado del sedimento marino en base a la $\mathrm{CI}_{50}$ para valores estadísticamente significativos.

\begin{tabular}{|ll}
\hline CI50 $(\boldsymbol{\%})$ & Nivel de toxicidad \\
\hline $90-100$ & No toxicidad \\
$82-89$ & Baja toxicidad \\
$59-81$ & Moderada toxicidad \\
$<59$ & Alta toxicidad \\
\hline
\end{tabular}

\section{Resultados y discusión}

Pruebas de sensibilidad con la microalga Isochrysis galbana

El crecimiento microalgal fue exponencial en las concentraciones $0.25 \mathrm{mg} / \mathrm{l}$ y $0.5 \mathrm{mg} / \mathrm{l} \mathrm{de} \mathrm{CuSO}_{4} \cdot 5 \mathrm{H}_{2} \mathrm{O}$ durante el tiempo que duró la prueba de toxicidad. El efecto inhibitorio de la microalga respecto al control se empezó a notar a partir de las concentraciones $1.0 \mathrm{mg} / \mathrm{l}$ y $2.0 \mathrm{mg} / 1 \mathrm{de} \mathrm{CuSO}_{4} \cdot 5 \mathrm{H}_{2} \mathrm{O}$ a las $96 \mathrm{~h}$ de exposición (Figura 2). Esta inhibición se debe a que los iones de cobre se adsorben en la membrana citoplasmática de las algas, deteniendo la división celular, tal como lo mencionan Liu et al. (2011). También se estimó la $\mathrm{CI}_{50}$ $=0.88 \mathrm{mg} / \mathrm{l} \mathrm{de} \mathrm{CuSO}_{4} \cdot 5 \mathrm{H}_{2} \mathrm{O}$ a las $96 \mathrm{~h}$ de exposición. Yap et al. (2004) obtuvieron un valor de $\mathrm{CI}_{50}=0.91$ $\mathrm{mg} / \mathrm{l}$ de $\mathrm{CuSO}_{4} \cdot 5 \mathrm{H}_{2} \mathrm{O}$ para I. galbana después de $120 \mathrm{~h}$ de exposición, siendo este valor cercano al hallado en el presente estudio a las 96 h de exposición.

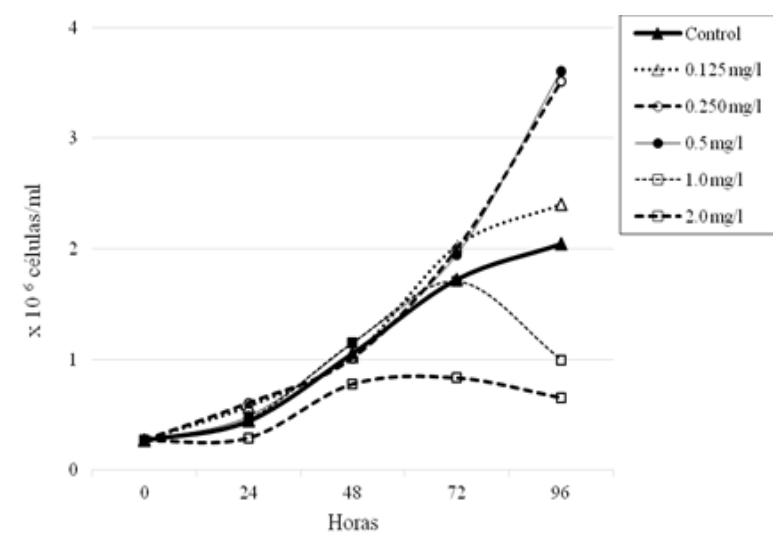

Figura 2. Crecimiento celular promedio (células $/ \mathrm{ml}$ ) de Isochrysis galbana expuesta a diferentes concentraciones de sulfato de cobre pentahidratado $\left(\mathrm{CuSO}_{4} \cdot 5 \mathrm{H}_{2} \mathrm{O}\right)$ a las $24,48,72$ y 96 h de exposición.

Pruebas de sensibilidad con la microalga Nannochloropsis oceanica

La tendencia de crecimiento microalgal en las concentraciones $\quad 0.125 \quad \mathrm{mg} / \mathrm{l} \quad \mathrm{y} \quad 0.250 \quad \mathrm{mg} / \mathrm{l} \quad \mathrm{de}$ $\mathrm{CuSO}_{4} \cdot 5 \mathrm{H}_{2} \mathrm{O}$ fue exponencial hasta las $96 \mathrm{~h}$ de exposición; mientras que en las concentraciones 0.5 $\mathrm{mg} / \mathrm{l}$ y $1.0 \mathrm{mg} / \mathrm{l}$ de $\mathrm{CuSO}_{4} \cdot 5 \mathrm{H}_{2} \mathrm{O}$ el crecimiento microalgal disminuyó respecto al control. Finalmente, en la concentración $2.0 \mathrm{mg} / \mathrm{l}$ de $\mathrm{CuSO}_{4} \cdot 5 \mathrm{H}_{2} \mathrm{O}$ el crecimiento algal se inhibió respecto al control desde las 24 horas (Figura 3). Además, el valor de $\mathrm{CI}_{50}$ obtenido a las $96 \mathrm{~h}$ de exposición fue de $1.44 \mathrm{mg} / \mathrm{l}$ de $\mathrm{CuSO}_{4} \cdot 5 \mathrm{H}_{2} \mathrm{O}$.

Se demostró que, la sensibilidad de la microalga I. galbana es mayor que la de $N$. oceanica al cabo de las 96 horas de exposición al sulfato de cobre pentahidratado $\left(\mathrm{CuSO}_{4} \cdot 5 \mathrm{H}_{2} \mathrm{O}\right)$, detectando valores de $\mathrm{CI}_{50}=0.88 \mathrm{mg} / 1$ y CI $\mathrm{C}_{50}=1.44 \mathrm{mg} / \mathrm{l}$, respectivamente. Moreno et al. (2000) obtuvieron valores de 0.0004 $\mathrm{mg} / \mathrm{l}$ para I. galbana y $0.0167 \mathrm{mg} / \mathrm{l}$ para otra especie de Nannochloropsis, N. atomus. Liu et al. (2011) mencionan que los mecanismos de toxicidad de cobre afectan la motilidad de las microalgas, la misma que está directamente relacionada con los procesos de respiración que proporcionan energía para la motilidad. Esta puede ser una razón por la que I. galbana es más 
sensible que $N$ oceanica, ya que cuenta con un par de flagelos, mientras que $N$. oceanica no tiene flagelos.

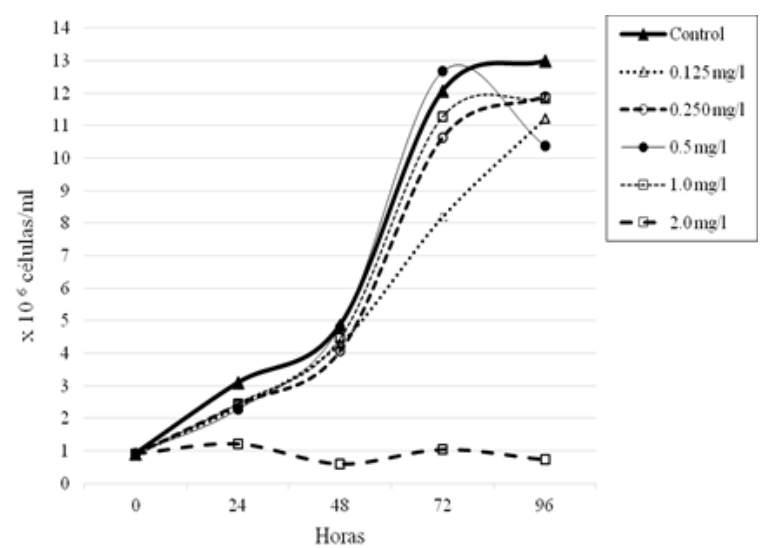

Figura 3. Crecimiento celular promedio (células/ml) de Nannochloropsis oceanica expuesta a diferentes concentraciones de sulfato de cobre a las 24, 48, 72 y 96 h de exposición.

$\underline{\text { Toxicidad del elutriado del sedimento marino en las }}$ bahías Los Chimus, Tortugas y Casma

En la fase preliminar de las pruebas de toxicidad efectuada el 8 (marzo 2016) y 10 (agosto 2016) para las estaciones de muestreo, se detectaron que tres estaciones del mes de marzo de 2016 (E1, E2 y E12) y dos estaciones de muestreo del mes de agosto 2016 (E2 y E10) pasaron a la fase definitiva o multiconcentración.

Siguiendo los valores propuestos por Bay et al. (2014), se estimó el nivel de toxicidad según la $\mathrm{CI}_{50}$. Las pruebas de toxicidad con la microalga I. galbana detectó distintos niveles de toxicidad, mientras que con $N$. oceanica se detectó "no toxicidad" para los elutriados de sedimento de las mismas estaciones de muestreo (Tabla 4).

En la Figura 4 se muestran los niveles de toxicidad de los elutriados de sedimentos en el área de estudio, observándose en la zona frente a Los Chimus "alta toxicidad" (E1) en marzo. En la estación E2 se presentó "moderada toxicidad" en marzo y agosto de 2016. La estación E10 en el mes de marzo de 2016 mostró un nivel de "no toxicidad", pero en agosto de 2016 se observó un nivel de "alta toxicidad". Finalmente, la estación E12 presentó "alta toxicidad" en marzo de 2016. Lofrano et al. (2017) mencionan que la toxicidad de los sedimentos costeros puede ser debido a la industria, el transporte $\mathrm{y}$ otras actividades antropogénicas.

Además, la propia actividad acuícola de la zona de estudio, especialmente en las bahías Los Chimus y Tortugas, podrían generar ciertos impactos negativos que contribuirían a la toxicidad del sedimento marino, como, por ejemplo, la producción de pseudoheces y heces, que es uno de los principales problemas de los cultivos de bivalvos por el impacto que genera en el medio, generando eutrofización, anoxia y sulfuros tóxicos produciendo la interrupción de los procesos bentónicos, problemas de toxicidad para los organismos en cultivo; debido a que la materia orgánica puede cambiar la demanda de oxígeno disuelto, produciendo alteraciones en los sedimentos, cambiando las condiciones redox y/o toxicidad creciente (Rice, 2008; Rudolph et al., 2009; Albarano et al., 2019).

Tabla 4. Concentración de Inhibición media $\left(\mathrm{CI}_{50}\right)$ de Isochrysis galbana y Nannochloropsis oceanica y nivel de toxicidad del elutriado de sedimento marino de las bahías Los Chimus, Tortugas y Casma, 2016.

\begin{tabular}{|c|c|c|c|c|c|}
\hline \multirow[b]{2}{*}{ : } & \multirow[b]{2}{*}{ 产 } & \multicolumn{2}{|c|}{ Isochrysis galbana } & \multicolumn{2}{|c|}{$\begin{array}{l}\text { Nannochloropsis } \\
\text { oceanica }\end{array}$} \\
\hline & & $\begin{array}{l}\mathrm{CI}_{50} \\
(\%)\end{array}$ & $\begin{array}{l}\text { Nivel de } \\
\text { toxicidad }\end{array}$ & $\begin{array}{l}\mathrm{CI}_{50} \\
(\%)\end{array}$ & $\begin{array}{l}\text { Nivel de } \\
\text { toxicidad }\end{array}$ \\
\hline \multirow{4}{*}{ 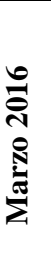 } & E1 & 4.30 & Alta toxicidad & $>100$ & No tóxico \\
\hline & E2 & 68.98 & Moderada & $>100$ & No tóxico \\
\hline & $\begin{array}{l}\text { E9, E10, } \\
\text { E11, } \\
\text { E14 }\end{array}$ & $>100$ & No tóxico & $>100$ & No tóxico \\
\hline & E12 & 7.97 & Alta toxicidad & $>100$ & No tóxico \\
\hline \multirow{4}{*}{ 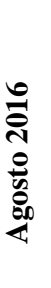 } & E13 & $>100$ & No tóxico & $>100$ & No tóxico \\
\hline & E2 & 75.31 & Moderada & $>100$ & No tóxico \\
\hline & $\begin{array}{l}\text { E3, E4, } \\
\text { E5, E6, } \\
\text { E7, E8, } \\
\text { E9, E14 }\end{array}$ & $>100$ & No tóxico & $>100$ & No tóxico \\
\hline & E10 & 15.36 & Alta toxicidad & $>100$ & No tóxico \\
\hline
\end{tabular}

La toxicidad de los sedimentos es esencial en los estudios de seguimiento para caracterizar el estado de los ambientes acuáticos y de esta manera tomar decisiones sobre áreas contaminadas (Albarano et al., 2019). Finalmente, de acuerdo con la sensibilidad observada en el presente estudio de investigación de la microalga I. galbana para detectar diferentes niveles de toxicidad de sedimentos marinos, podría emplearse para evaluar esta matriz ambiental como complemento a pruebas químicas, ya que no se discrimina el origen de la toxicidad. Además, la información obtenida afirma que existe toxicidad de sedimentos marinos en las bahías Los Chimus, Tortugas y Casma al exponerlos a la microalga I. galbana, por lo que se recomienda seguir evaluando el sedimento marino de estas zonas de estudio con el fin de asegurar la sostenibilidad de la maricultura y la pesca artesanal. 


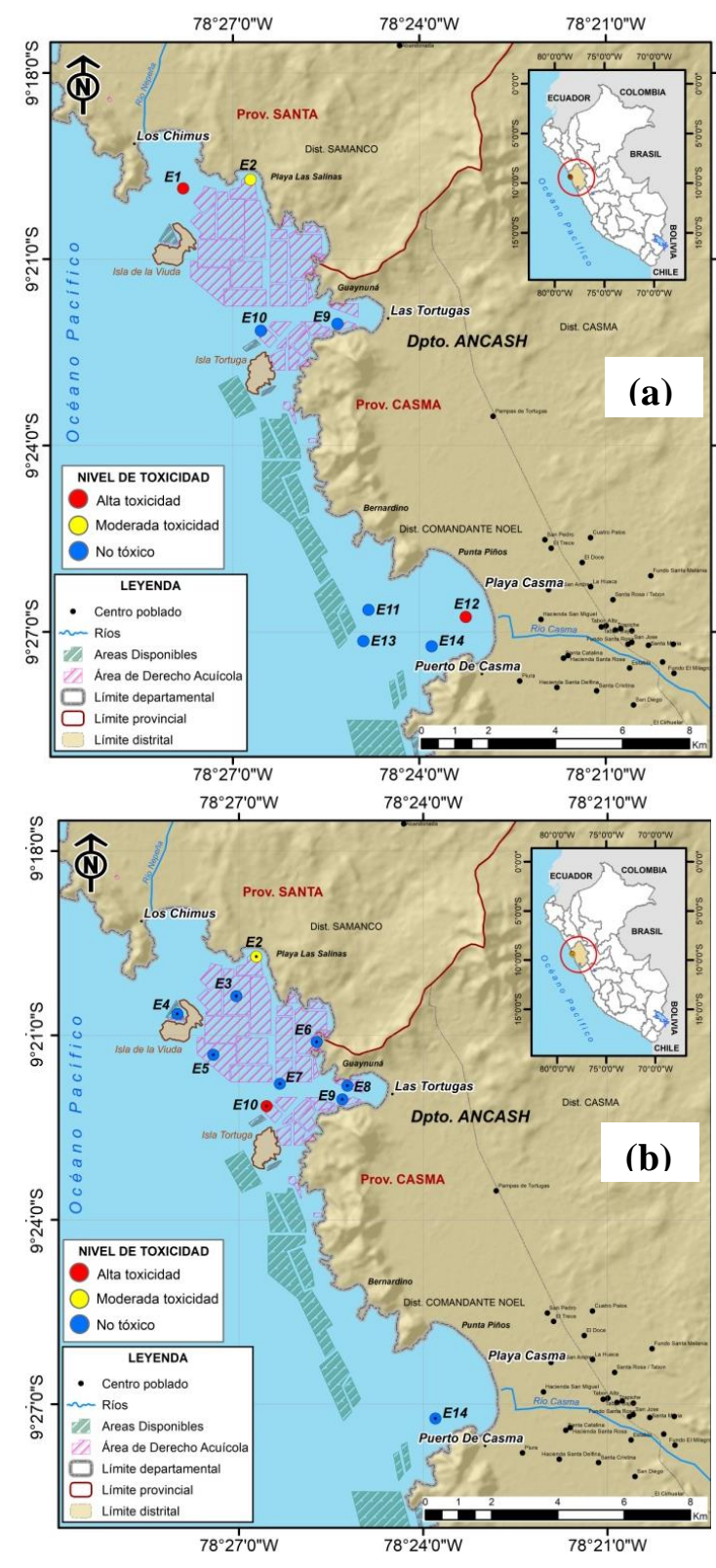

Figura 4. Niveles de toxicidad del sedimento marino de las bahías Los Chimus, Tortugas y Casma en Áncash (Perú) empleando la microalga Isochrysis galbana. (a) marzo 2016 y (b) agosto 2016.

\section{Conclusiones}

Se demostró que la sensibilidad de la microalga I. galbana es mayor que la de $N$. oceanica al cabo de las 96 horas de exposición al tóxico de referencia, sulfato de cobre pentahidratado $\left(\mathrm{CuSO}_{4} \cdot 5 \mathrm{H}_{2} \mathrm{O}\right)$, detectando valores de $\mathrm{CI}_{50}=0.88 \mathrm{mg} / \mathrm{l}$ y $\mathrm{CI}_{50}=1.44$ $\mathrm{mg} / \mathrm{l}$, respectivamente. La causa posible de esta sensibilidad es por la presencia de flagelos que le sirven para motilidad a I. galbana. Los elutriados de los sedimentos marinos provenientes de las bahías Los Chimus, Tortugas y Casma, bajo las condiciones experimentales de este trabajo de investigación, presentaron diferentes niveles de toxicidad "moderada toxicidad" $\left(\mathrm{CI}_{50-96}=59 \%\right.$ - 81\%) y "alta toxicidad" $\left(\mathrm{CI}_{50-96}<59 \%\right)$ al exponer a la microalga I. galbana, encontrándose los valores de $\mathrm{CI}_{50}$ entre $4.30 \%$ y $68.98 \%$, mientras que la microalga $N$. oceanica mostró "no toxicidad" frente a las mismas muestras de elutriados de sedimentos. Por último, las pruebas de toxicidad demuestran que la microalga I. galbana detecta diferentes niveles de toxicidad, por lo que se podría emplear para evaluar ambientalmente muestras de sedimento marino, como complemento a pruebas químicas ya que no se discrimina el origen de la toxicidad.

\section{Agradecimientos}

Nuestro agradecimiento al Proyecto por Resultado "Evaluación del estado, presión y respuesta ambiental en la bahía Tortugas-Casma" del Instituto del Mar del Perú. A Aida Henostroza, Manuel Mendoza y Ricardo Dioses del Laboratorio de Ecotoxicología acuática del Instituto del Mar del Perú, por su apoyo durante la evaluación de las pruebas de toxicidad.

\section{Literatura citada}

Alayo M., Iannacone J. \& Arrascue A. 2004. Sensibilidad al cromo: Microbiopruebas con las diatomeas marinas Isochrysis galbana Parke y Chaetoceros gracilis Schütt. Ecología Aplicada, 3(1,2): 154-161. DOI: http://dx.doi.org/10.21704/rea.v3i1-2.285.

Albarano L., Costantini M., Zupo V., Lofrano G., Guida M. \& Libralato G. 2019. Marine sediment toxicity: A focus on micro and mesocosms towards remediation Science of The Total Environment, 708: 134837. DOI: https://doi.org/10.1016/j.scitotenv.2019.134837.

Alzahrani D.A., Selim E.M.M. \& El-Sherbiny M. 2018. Ecological assessment of heavy metals in the grey mangrove (Avicennia marina) and associated sediments along the Red Sea coast of Saudi Arabia. Oceanologia, 60(4): 513-526. DOI: https://doi.org/10.1016/j.oceano.2018.04.002.

Bay S., Greenstein D., Diehl D. \& Fetscher A. 2014. Sediment quality assessment technical support manual. Southern California Coastal wáter research Project. Technical report 777 - January 2014.

Díaz M., Bustos M. \& Espinosa A. 2014. Pruebas de toxicidad acuática: fundamentos y métodos. Primera edición. Universidad Nacional de Colombia. Bogotá, Colombia.

García V. 2016. Evaluación del estado y presión ambiental en el litoral costero de Los Chimus a Casma. Región Áncash. Informe Interno. Instituto del Mar del Perú.

García V., Perea de la Matta Á. \& Orozco M. 2018. Calidad del ambiente marino y costero en la Región Áncash, 2018. Bol. Inst. Mar Perú, 34(2): 406-431. URI: https://hdl.handle.net/20.500.12958/3395. 
Giraldo A., Montes R., Rodil R., Quintana J., Vidal L. \& Beiras R. 2017. Ecotoxicological Evaluation of the UV Filters Ethylhexyl Dimethyl p-Aminobenzoic Acid and Octocrylene Using Marine Organisms Isochrysis galbana, Mytilus galloprovincialis and Paracentrotus lividus. Archives of Environmental Contamination and Toxicology , 72: 606-611. DOI: https://doi.org/10.1007/s00244-017-0399-4.

Hampel M., Moreno I., Sobrino C., Lubián L. \& Blasco J. 2001. Acute toxicity of LAS homologues in marine microalgae: Esterase activity and inhibition growth as endpoints of toxicity. Ecotoxicology and Environmental Safety, 48(3): 287-292. DOI: https://doi.org/10.1006/eesa.2000.2028.

IMARPE. 2006. Identificación y delimitación de bancos naturales de invertebrados marinos, zonas de pesca artesanal y áreas propuestas para la maricultura en el Litotal de la Región Ancash. Inf. Inst. Mar Perú 33(4). IMARPE (Instituto del Mar del Perú). Callao-Perú. URI: http://biblioimarpe.imarpe.gob.pe/handle/1234567 89/1948.

Liu G., Chai X., Shao Y., Hu L., Xie Q. \& Wu H. 2011. Toxicity of copper, lead, and cadmium on the motility of two marine microalgae Isochrysis galbana and Tetraselmis chui. Journal of Environmental Sciences, 23(2): 330-335. DOI: https://doi.org/10.1016/S10010742(10)60410-X.

Lofrano G., Libralato G., Minetto D., De Gisi S., Todaro F., Conte B., Calabrò D., Quatraro L. \& Notarnicola M. 2017. In situ remediation of contaminated marinesediment: an overview. Environmental Science and Pollution Research, 24(6): 5189-5206. DOI: https://doi.org/10.1007/s11356-016-8281-x.

MacIntosh A., Dafforn K., Penrose B., Chariton A. \& Cresswell T. 2021. Ecotoxicological effects of decommissioning offshore petroleum infrastructure: A systematic review. Critical Reviews in Environmental Science and Technology, 07 May [online]. DOI: https://doi.org/10.1080/10643389.2021.1917949.

Manz W., Krebs F., Scgipper C. \& Den Besten P. 2007. Status of ecotoxicological assessment of sediment and dredged material in Germany and The Netherlands. DutchGerman Exchange (DGE) on Dredged Material - Part 5 . Federal Institute of Hydrology (BfG). Germany. URI: https://sednet.org/download/DGE-Report-5Ecotoxicology.pdf.

Maranho L., Garrido M., DelValls T. \& Martín-Díaz M. 2015 Suitability of Standardized Acute Toxicity Tests for Marine Sediment Assessment: Pharmaceutical Contamination.Water, Air \& Soil Pollution, 226: 65. DOI: http://dx.doi.org/10.1007/s11270-014-2273-6.

Moreno I., Lubián L. \& Soares A. 2000. Influence of celular density on determination of $\mathrm{EC}_{50}$ in microalgal growth inhibition tests. Ecotox and environ saf, 47: 112- 116. DOI:_https://doi.org/10.1006/eesa.2000.1953.

Otero A., Cruz P. \& Velasco Y. 2013. Evaluación del efecto del hidrocarburo fenantreno sobre el crecimiento de Chlorella vulgaris (CHLORELLACEAE). Acta Biológica Colombiana, 18(1): 87-98. https://revistas.unal.edu.co/index.php/actabiol/article/vie $\mathrm{w} / 24558$.
Paredes C. 2018. Evaluación del riesgo ecológico de la barita (BaSO4) empleando pruebas ecotoxicológicas con doce organismos. Ecol. apl., 17(1): 23-37. DOI: http://dx.doi.org/10.21704/rea.v17i1.1170.

Regidor H. \& Guzmán J. 2015. Toxicidad del agua de producción hidrocarburífera sobre el pez astyanax abramis (jenyns 1842). Multiciencias, 15(2): 143-148. http://www.redalyc.org/articulo.oa?id=90443048002.

Rice M. 2008. Environmental Effects of Shellfish Aquaculture in the Northeast. NRAC (Northeastern Regional Acuaculture Center), Publication No. 105-2008. University of Maryland. https://web.uri.edu/coopext/files/Environmental-Effectsof-Shellfish.pdf.

Rudolph A., Medina P., Urrutia C. \& Ahumada R. 2009. Ecotoxicological sediment evaluations in marine aquaculture areas of Chile. Environ. Monit. Assess., 155: 419-429. DOI: https://doi.org/10.1007/s10661-0080444-x.

Rudolph A., Medina P., Novoa V. Ahumada R. \& Cortés I. 2011. Calidad ecotoxicológica de los sedimentos en fiordos del sur de Chile. Rev. biol. mar. oceanogr. 46(1): 79-84. DOI: https://dx.doi.org/10.4067/S071819572011000100012.

Rudolph A., Novoa V. Ahumada R., Saez K. \& Silva N. 2014. Calidad de los sedimentos marinos entre el estrecho de Magallanes y el cabo de hornos (Patagonia Chilena) en function de pruebas de toxicidad. Rev. biol. mar. oceanogr., DOI: http://dx.doi.org/10.4067/S071819572014000100002.

Simpson S., Batley G., Chariton A., Stauber J., Rey C., Chapman J., Hyne R., Gale S., Roach C. \& Maher W. 2005. Manual para la Evaluación de la Calidad de Sedimentos. CSIRO: Bangor, Nueva Gales del Sur.

Trenfield M., van Dam J., Harford A., Parry D., Streten C., Gibb K. \& Van Dam R. 2015. Aluminium, Gallium and molybdenum toxicity the tropical marine microalga Isochrysis galbana. Environmental Toxicology and Chemistry, 34(8): 1833-1840. DOI: http://dx.doi.org/10.1002/etc.2996.

Uribe R., Perea A., García V. \& Huerto M. 2019. Biodiversidad marina en el norcentro de la costa de Peru: Un enfoque para la evaluación de planes de manejo. Bol. Inst. Mar Perú, 34(2): 332-350.URI: https://hdl.handle.net/20.500.12958/3401.

USEPA \& USACE. 1998. Evaluation of Dredged Material Proposed for Discharge in Waters of the U.S. - Testing Manual. Inland Testing Manual. EPA (United States Environmental Protection Agency), EPA-823-B-98-004. USACE (United States Army Corps of Engineers). Washington.

https://www.epa.gov/sites/production/files/201508/docu ments/inland_testing_manual_0.pdf.

USEPA. 1992. Sediment classification methods compendium. EPA (United States Environmental Protection Agency), EPA 823-R-92-006. Washington DC.

https://archive.epa.gov/water/archive/web/pdf/classmeth ods.pdf. 
USEPA. 1994. Methods for assessing the toxicity of sediment-associated contaminants with estuarine and marine amphipods. EPA (United States Environmental Protection Agency), EPA/600/R-94/025 https://archive.epa.gov/water/archive/web/pdf/marineme thod.pdf.

USEPA. 2002. Short-term Methods for Estimating the Chronic Toxicity of Effluents and Receiving Waters to Marine and Estuarine Organisms. Third Edition. EPA (United States Environmental Protection Agency), EPA821-R-02-014.

https://www.epa.gov/sites/production/files/2015-

08/documents/short-term-chronic-marine-and-estuarinewet-manual_2002.pdf.

USEPA. 2012. Ecological Effects Test Guidelines OCSPP 850.4500: Algal Toxicity. EPA (United States Environmental Protection Agency), EPA 712-C-006. https://nepis.epa.gov/Exe/ZyPDF.cgi/P100J6UE.PDF?D ockey=P100J6UE.PDF.

Valdés J. \& Castillo A. 2014. Evaluación de la calidad ambiental de los sedimentos marinos en el sistema de bahías de Caldera $\left(27^{\circ} \mathrm{S}\right)$, Chile. Lat. Am. J. Aquat. Res., 42(3):497-513.

http://www.lajar.cl/index.php/rlajar/article/view/vol42issue3-fulltext-10/461.

Velázquez L.M. 2010. Evaluación del efecto de la exposición al hidrocarburo benzo(a)pireno sobre la eficiencia fotosintética de tres microalgas marinas.Tesis de Maestría en Ciencias. CICESE (Centro de Investigación Científica y de Educación Superior de Ensenada). Baja California / México.

http://cicese.repositorioinstitucional.mx/jspui/handle/10 07/2583.
Vera G., Tam J., Pinto E. \& Angulo J. 2001. Efecto del cadmio sobre el crecimiento poblacional de la Diatomea marina Chaetoceros gracilis Schutt. Revista peruana de Biología, 8(1): 45-52. DOI: https://doi.org/10.15381/rpb.v8i1.6512.

Visviki I. \& Rachlin J. 1992. Ultrastructural changes in Dunaliella minuta following acute and chronic exposure to copper and cadmium. Arch. Environ. Contam. Toxicol., 23: 420-425. DOI: https://doi.org/10.1007/BF00203803.

Wong P. \& Dixon D. 1995. Bioassessment of wáter Quality. Environmental Toxicology and Water Quality, 10(1): 917. DOI: https://doi.org/10.1002/tox.2530100103.

Yap C., Ismail A., Omar H. \& Tan S. 2004. Toxicities and tolerances of $\mathrm{Cd}, \mathrm{Cu}, \mathrm{Pb}$ and $\mathrm{Zn}$ in a primary producer (Isochrysis galbana) and in a primary consumer (Perna viridis). Environment International, 29(8): 10971104. DOI: https://doi.org/10.1016/S01604120(03)00141-7.

Ynga G. \& Niño A. 2019. Manual para producción de microalgas marinas. INFORME, 46(1): 5-16. Inst. Mar Perú. URI: https://hdl.handle.net/20.500.12958/3338.

Zar J.GH. 1996. Bioestatistical analysis. Prentice-Hall. 3ed. New York, USA.

\footnotetext{
${ }^{1}$ Facultad de Pesquería / Universidad Nacional Agraria La Molina (UNALM). Lima / Perú.

2 hlopez_cabanillas@hotmail.com.

${ }^{3}$ mcmiglio@lamolina.edu.pe.

${ }^{4}$ Organismo de Evaluación y Fiscalización Ambiental (OEFA). cparedes1076@gmail.com.
} 